\title{
GENERALIZED CONTINUUM OVERALL MODELLING OF PERIODIC COMPOSITE STRUCTURES
}

\author{
Duy Khanh Trinh, Samuel Forest \\ MINES ParisTech Centre des matériaux, \\ CNRS UMR 7633 BP 87, F-91003 Evry Cedex, France
}

\begin{abstract}
Classical homogenization methods fail to reproduce the overall response of composite structures when macroscopic strain gradients become significant. Generalized continuum models like Cosserat, strain gradient and micromorphic media, can be used to enhance the overall description of heterogeneous materials when the hypothesis of scale separation is not fulfilled. We show in the present work how the higher order elasticity moduli can be identified from suitable loading conditions applied to the unit cell of a periodic composite. The obtained homogeneous substitution generalized continuum is used then to predict the response of a composite structure subjected to various loading conditions. Reference finite element computations are performed on the structure taking all the heterogeneities into account. The overall substitution medium is shown to provide improved predictions compared to standard homogenization. In particular the additional boundary conditions required by generalized continua makes it possible to better represent the clamping conditions on the real structure.
\end{abstract}

Keywords: Homogenization, strain gradient effects, periodic micro-structures, numerical prediction.

\section{INTRODUCTION}

Computational analysis of composite structures is based on the consideration of homogeneous effective behaviour of the heterogeneous material. Such effective properties can be deduced from the knowledge of the detailed arrangement of the constituents by means of homogenization methods, as depicted in [24]. Classical homogenization methods root on the assumption of separation of scales, meaning that the size of the heterogeneities must be significantly smaller than the minimal structure size, or, more precisely, than the smallest wave length of the applied loading conditions. Although this condition is usually met for standard plies made of fiber composites or for particle composites, the condition fails in the case of some architectured materials which contain a finite number of millimeter size unit cells. Individual cells are then subjected not only to homogeneous overall loading but also to significant overall strain gradients. Even though the homogeneous equivalent medium (HEM) constructed by means of classical homogenization techniques, very often does a good job at the limit of scale separation [20], some remedy is necessary in general to improve the performance of the HEM compared to the actual response of the heterogeneous 
material. This is especially the case at boundaries where standard boundary conditions fail to account for the actual clamping or more complex conditions. Separation of scales breaks down for example in sandwich composites when the core material is made of finite sized structural elements like honeycombs or trusses.

In the last 15 years, it has been proposed to resort to the mechanics of generalized continua which incorporate additional degrees of freedom or higher order strain gradient terms, see for instance $[10,13,16,17,18,19,21]$. Higher order homogenization schemes have been proposed to replace the composite material by a homogeneous substitution generalized medium. The different strategies are sketched in Fig. 1 where the flux (1)

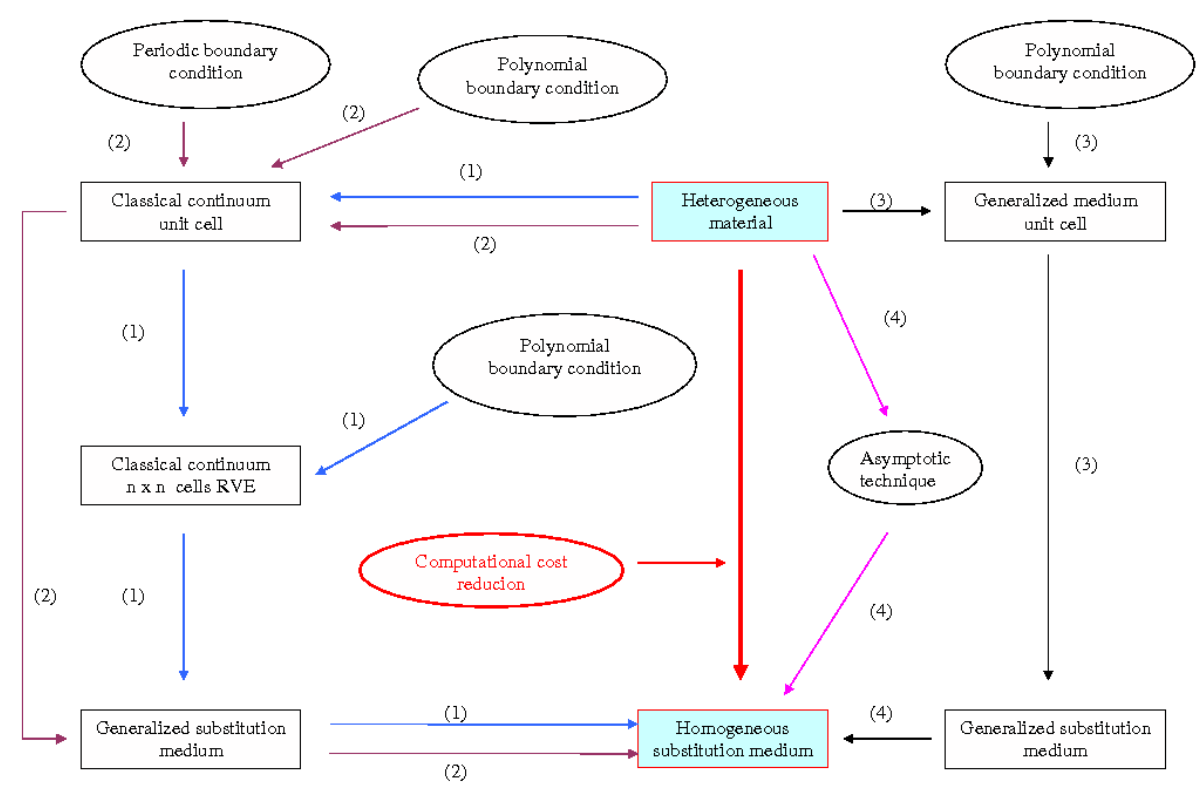

Fig. 1. Different generalized continuum homogenization strategies

denotes the strategy of replacing a periodic microstructure by a homogeneous generalized continuum model. It has to be distinguished from other contributions like $[9,22,28]$, in which both the heterogeneous material and homogeneous substitution medium are modelled by generalized continua. These contributions are counted in flux (3) of Fig. 1. In [4], a multiscale asymptotic expansion was proposed to identify higher order strain gradient contributions to the overall response. It represents a systematic method to compute correctors for overcoming the mentioned limitations of classical homogenization. But the method remains complex and difficult to extend to non-linear mechanical behaviour. This research direction is counted in flux (4) of Fig. 1. In contrast, higher-order homogenization based on polynomial expansion of the displacement field can be applied to non-linear material as in $[6,8,14,19,27]$. Higher-order homogenization, initially proposed in $[5,7,11,15,20]$, is an extension of usual affine conditions of loading in classical homogenization in order to incorporate strain gradient effects in the homogenization procedure. Such polynomial 
developments represent an alternative to multiscale asymptotic expansions to derive effective higher order properties, with the advantage that they can be used in a straightforward manner, irrespective of the local linear or nonlinear behaviour of the composite material. The complete Ansatz polynomial, introduced for instance in [12], is

$$
u_{i}^{*}(\underline{\boldsymbol{x}})=E_{i j} x_{j}+\frac{1}{2} D_{i j k} x_{j} x_{k}+\frac{1}{3} D_{i j k l} x_{j} x_{k} x_{l}+\frac{1}{4} D_{i j k l m} x_{j} x_{k} x_{l} x_{m}
$$

where $u_{i}$ are the components of the displacement vector of material points with coordinates $x_{i}$ belonging to the unit cell and the $D_{i j / i j k / i j k l / i j k l m}$ are the coefficients of the polynomials. Some quadratic and third order terms of this polynomials were selected for the construction of a homogeneous orthotropic Cosserat substitution medium in [10]:

$$
\begin{aligned}
& u_{1}^{*}=E_{11} x_{1}+E_{12} x_{2}-K_{31} x_{1} x_{2}-\frac{K_{32}}{2} x_{2}^{2}+\hat{D}\left(x_{2}^{3}-3 x_{1}^{2} x_{2}\right) \\
& u_{2}^{*}=E_{12} x_{1}+E_{22} x_{2}+K_{32} x_{1} x_{2}+\frac{K_{31}}{2} x_{1}^{2}-\hat{D}\left(x_{1}^{3}-3 x_{1}^{2} x_{2}\right)
\end{aligned}
$$

in the two - dimensional case. Such a polynomial field generally does not lead to an equilibrated stress field inside the unit cell so that a fluctuation vector field $\underline{\boldsymbol{v}}$ must exist in the form:

$$
\underline{\boldsymbol{u}}(\underline{\boldsymbol{x}})=\underline{\boldsymbol{u}}^{*}(\underline{\boldsymbol{x}})+\underline{\boldsymbol{v}}(\underline{\boldsymbol{x}})
$$

When the quadratic and cubic coefficients vanish, the fluctuation must be periodic in a composite with periodic microstructure, as required by classical homogenization [24]. This is no longer the case when higher order terms are included. The fact that $\underline{\boldsymbol{v}}$ can generally not be expected to be periodic was first discussed in $[29]$ and proved in $[12,26]$ by determining a representative volume element size for quadratic and cubic boundary conditions.

This methodology was recently applied to a simple two - phase periodic microstructure in [25] and shown to deliver better description of the displacement field in a loaded composite structure than the usual Cauchy approach. In particular, the effective Cosserat and second gradient higher order elastic moduli were determined using finite element simulations on the unit cell. The objective of the present work is to compare reference computations of composite structures including all heterogeneities, to the responses of homogenization substitution media like Cosserat and second gradient continua. The latter responses will be determined either analytically or by finite element simulations. We will validate the approach in one loading case but also show its limitations in another one.

The work is presented in the context of small deformations. A Cartesian orthonormal basis is used throughout the work. Regarding notations, first, second and third order tensors are denoted $\underline{\boldsymbol{a}}, \underset{\sim}{\boldsymbol{a}}$ and $\underset{\sim}{\boldsymbol{a}}$, respectively. Fourth and sixth order tensors, $\underset{\approx}{\boldsymbol{a}}$ and $\underset{\approx}{\boldsymbol{a}}$ will also be necessary. Single, double and triple contractions are written:

$$
\underline{\boldsymbol{a}} \cdot \underline{\boldsymbol{b}}=a_{i} b_{i}, \quad \underset{\sim}{\boldsymbol{a}}: \underset{\sim}{\boldsymbol{b}}=a_{i j} b_{i j}, \quad \underset{\sim}{\boldsymbol{a}}: \underset{\sim}{\boldsymbol{a}}=a_{i j k} a_{i j k}
$$

Partial derivative of a component $a$ with respect to coordinate $j$ is denoted by $a_{, j}$. 


\section{GENERALIZED CONTINUA AND HIGHER ORDER HOMOGENIZATION}

\subsection{Cosserat overall modeling of composites}

The macroscopic homogeneous substitution medium is assumed to be adequately represented by a Cosserat continuum characterized by the macroscopic displacements $\underline{\boldsymbol{U}}$ and the Cosserat microrotation vector $\boldsymbol{\Phi}$. Following the homogenization scheme proposed in [11], the macroscopic degrees of freedom are defined as statistical moments of the distribution of the local displacement field inside a two - dimensional square unit cell $V$ with edge length $l$ :

$$
\underline{\boldsymbol{U}}(\underline{\boldsymbol{X}})=<\underline{\boldsymbol{u}}(\underline{\boldsymbol{x}})>, \quad \underline{\boldsymbol{\Phi}}=\frac{6}{l^{2}}<(\underline{\boldsymbol{x}}-\underline{\boldsymbol{X}}) \times \underline{\boldsymbol{u}}>
$$

The brackets $<\cdot>$ denote volume averaging over the unit cell $V$. The macroscopic coordinate $\underline{\boldsymbol{X}}$ is the center of the unit cell $V$.

The Cosserat macro - deformation $\underset{\sim}{\boldsymbol{e}}$ and curvature tensor $\underset{\sim}{\boldsymbol{K}}$ are defined as:

$$
e_{i j}=U_{i, j}+\epsilon_{i j k} \Phi_{k}, \quad K_{i j}=\Phi_{i j}
$$

where $\underset{\sim}{\boldsymbol{\epsilon}}$ is the permutation tensor. The stress tensors conjugate to the latter strain measures in the work of internal forces are the generally non - symmetric simple force stress tensor $\underset{\sim}{\boldsymbol{N}}$ and the couple stress tensor $\boldsymbol{M}$. They must fulfill the balance of momentum and balance of moment of momentum equations:

$$
\Sigma_{i j, j}=0, \quad M_{i j, j}-\epsilon_{i j k} \Sigma_{j k}=0
$$

in the absence of body forces. Generalized stress and strain tensors are related by the matrix of elastic moduli, written here in the two - dimensional plane strain case:

$$
\left[\begin{array}{c}
\Sigma_{11} \\
\Sigma_{22} \\
\Sigma_{12} \\
\Sigma_{21} \\
M_{31} \\
M_{32}
\end{array}\right]=\left[\begin{array}{cccccc}
Y_{1111} & Y_{1122} & 0 & 0 & 0 & 0 \\
Y_{1122} & Y_{2222} & 0 & 0 & 0 & 0 \\
0 & 0 & Y_{1212} & Y_{1221} & 0 & 0 \\
0 & 0 & Y_{1221} & Y_{2121} & 0 & 0 \\
0 & 0 & 0 & 0 & C_{3131} & 0 \\
0 & 0 & 0 & 0 & 0 & C_{3232}
\end{array}\right]\left[\begin{array}{l}
e_{11} \\
e_{22} \\
e_{12} \\
e_{21} \\
K_{31} \\
K_{32}
\end{array}\right]
$$

where the effective material was assumed to display orthotropic symmetry. The main objective of generalized continuum homogenization is to determine the elasticity moduli $Y_{i j k l}$ and $C_{i j k l}$ from the knowledge of the geometry of the periodic unit cell and the knowledge of the classical elasticity moduli of the constituents. In the $2 \mathrm{D}$ orthotropic Cosserat case, 8 moduli must be determined.

\subsection{Second gradient overall modeling of composites}

If the homogeneous substitution medium is regarded as a second gradient continuum, we introduce the second gradient of the displacement field in addition to the first gradient [23]:

$$
K_{i j k}=U_{i, j k}=<u_{i, j k}>
$$

It coincides with the mean value of the second gradient of the local displacement field inside the unit cell in the absence of holes, cracks nor rigid particles [12]. The conjugate 
quantity in the work of internal forces is the third rank tensor of double stress or hyper - stress tensor $M_{i j k}$. The simple and double stress tensors fulfill one single balance of momentum equation:

$$
\Sigma_{i j, j}-M_{i j k, j k}=0
$$

The simple stress and strain tensors are related by the usual fourth rank tensor of elasticity moduli. In addition to that, there exists a sixth rank tensor of higher order moduli which relates the second gradient of the displacement to the hyper-stress. In the orthotropic case, these relations read [25]:

$$
\left[\begin{array}{c}
M_{111} \\
M_{122} \\
\sqrt{2} M_{212} \\
M_{222} \\
M_{211} \\
\sqrt{2} M_{121}
\end{array}\right]=\left[\begin{array}{cccccc}
A_{11} & A_{12} & A_{13} & 0 & 0 & 0 \\
A_{12} & A_{22} & A_{23} & 0 & 0 & 0 \\
A_{13} & A_{23} & A_{33} & 0 & 0 & 0 \\
0 & 0 & 0 & A_{44} & A_{45} & A_{46} \\
0 & 0 & 0 & A_{45} & A_{55} & A_{56} \\
0 & 0 & 0 & A_{46} & A_{56} & A_{66}
\end{array}\right]\left[\begin{array}{c}
K_{111} \\
K_{122} \\
\sqrt{2} K_{212} \\
K_{222} \\
K_{211} \\
\sqrt{2} K_{121}
\end{array}\right]
$$

This makes 12 independent double elasticity moduli to be identified from the analysis of the response of the unit cell to non-homogeneous loading conditions.

\section{SOLUTION OF A DOUBLE SHEAR PROBLEM FOR COSSERAT AND SECOND GRADIENT CONTINUA}

A boundary value problem is solved for an orthotropic linear elastic Cosserat continuum. The considered problem was solved for an orthotropic second gradient medium by [2]. This solution will serve as a basis for the validation of the generalized homogenization approach in the next sections.
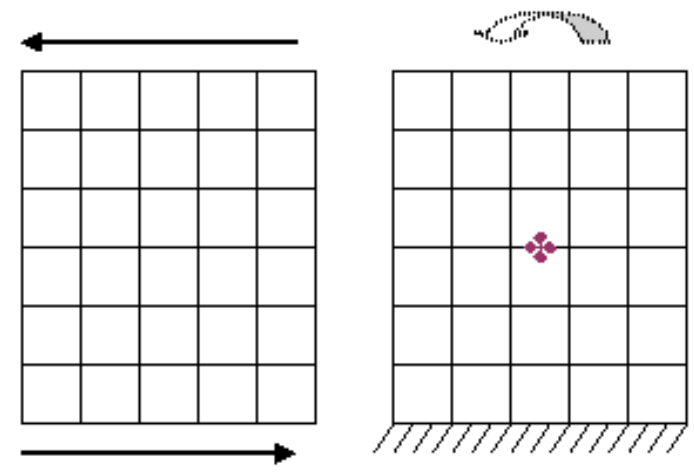

Fig. 2. Two loading conditions for a composite structure: Double shear conditions (left) and combined bending - shear conditions (right)

We consider a strip of homogeneous Cosserat material, infinite in the horizontal direction 1 , and with thickness $2 h$. The origin of the coordinate system is at the middle of the strip, see Fig. 2 (left). The following boundary conditions are applied:

$$
U_{1}(h)=\frac{\delta}{2}, \quad U_{2}(h)=0, \quad \Phi(h)=0, \quad U_{1}(-h)=-\frac{\delta}{2}, \quad U_{2}(-h)=0, \quad \Phi(-h)=0
$$


where $\Phi:=\Phi_{3}$ is the single activated microrotation component. The two unknown fields of the problem are $u(x):=U_{1}\left(x_{2}\right)$ and $\Phi\left(x_{2}\right)$. The deformation and curvature tensors are calculated as

$$
[\boldsymbol{\sim}]=\left[\begin{array}{ccc}
0 & u^{\prime}+\Phi & 0 \\
-\Phi & 0 & 0 \\
0 & 0 & 0
\end{array}\right], \quad[\underset{\sim}{\boldsymbol{K}}]=\left[\begin{array}{ccc}
0 & 0 & 0 \\
0 & 0 & 0 \\
0 & \Phi^{\prime} & 0
\end{array}\right]
$$

The constitutive relations (9) provide the force stress and couple stress components:

$$
\begin{aligned}
\Sigma_{12} & =Y_{1212} e_{12}+Y_{1221} e_{21}=Y_{1212}\left(u^{\prime}+\Phi\right)-Y_{1221} \Phi \\
\Sigma_{21} & =Y_{1221} e_{12}+Y_{2121} e_{21}=Y_{1221}\left(u^{\prime}+\Phi\right)-Y_{2121} \Phi \\
M_{32} & =C_{3232} K_{32}=C_{3232} \Phi^{\prime}
\end{aligned}
$$

The balance equations (8) provide the two following coupled differential equations:

$$
\begin{gathered}
Y_{1212}\left(u^{\prime \prime}+\Phi^{\prime}\right)-Y_{1221} \Phi^{\prime}=0 \\
C_{3232} \Phi^{\prime}-Y_{1212}\left(u^{\prime}+\Phi\right)+Y_{1221} \Phi+Y_{1221}\left(u^{\prime}+\Phi\right)-Y_{2121} \Phi=0
\end{gathered}
$$

Elimination of the displacement component $u$ in the previous equations leads to the following differential equation for the micro-rotation component:

$$
\Phi^{\prime \prime \prime}=\omega^{2} \Phi^{\prime} \quad \text { with } \quad \omega^{2}=\frac{Y_{1212} Y_{2121}-Y_{1221}^{2}}{Y_{1212} C_{3232}}
$$

The positivity of $\omega^{2}$ is ensured by the positivity of the elastic quadratic potential associated with the generalized Hooke law (9). It follows that the micro-rotation field is of the form:

$$
\Phi\left(x_{2}\right)=\frac{1}{\omega}\left(C_{1} \sinh (\omega x)+C_{2} \cosh (\omega x)\right)+C_{3}
$$

where the $C_{i}$ are integration constants. The boundary conditions (13) state that the microrotation should vanish at the upper and lower boundaries:

$$
\frac{1}{\omega}\left(C_{1} \sinh (\omega h)+C_{2} \cosh (\omega h)\right)+C_{3}=0, \quad \frac{1}{\omega}\left(-C_{1} \sinh (\omega h)+C_{2} \cosh (\omega h)\right)+C_{3}=0
$$

which requires

$$
C_{1}=0, \quad C_{3}=-\frac{1}{\omega} C_{2} \cosh (\omega h) \quad \Longrightarrow \quad \Phi\left(x_{2}\right)=\frac{1}{\omega} C_{2}(\cosh (\omega x)-\cosh (\omega h))
$$

The displacement field is obtained from the integration of equation (19)

$$
u=\left(\beta+\frac{\gamma}{\omega^{2}}\right) C_{2} \sinh (\omega x)+\gamma C_{3} x+C_{4}
$$

with

$$
\beta:=\frac{C_{3232}}{Y_{1221}-Y_{1212}}, \quad \gamma:=\frac{Y_{1212}-2 Y_{1221}+Y_{2121}}{Y_{1212}-Y_{1221}}
$$

The displacement boundary conditions (13) imply that

$$
C_{4}=0, \quad C_{2}=\frac{\delta}{2\left[\left(\beta+\frac{\gamma}{\omega^{2}}\right) \sinh (\omega x)-\frac{\gamma h}{\omega} \cosh (\omega h)\right]} .
$$


The same boundary value problem was solved for an orthotropic second gradient continuum by [2]. Their result is recalled here:

$$
U_{\beta}\left(y_{\alpha}\right)=A \cosh \left(\frac{y_{\alpha}}{\lambda_{S h-\alpha}}\right)+B \sinh \left(\frac{y_{\alpha}}{\lambda_{S h-\alpha}}\right)+C u_{\alpha}+D
$$

where $U_{\beta}$ is the non-vanishing component of displacement boundary condition and $\alpha$ is the perpendicular direction, $\frac{y_{\alpha}}{\lambda_{S h-\alpha}}=\sqrt{\frac{A_{\beta \alpha \alpha \beta \alpha \alpha}}{C_{\alpha \beta \alpha \beta}}}$ and the constants $A, B, C, D$ are determined from the boundary conditions. In this double shear problem, the constant are determined, and (27) returns:

$$
u(x)=\frac{\delta}{2 \omega^{2}\left[\frac{\sinh (\omega h)}{\omega^{2}}-\frac{h}{\omega} \cosh (\omega h)\right]} \sinh (\omega x)-\frac{C_{2}}{\omega} \cosh (\omega h) x
$$

where

$$
C_{2}=\frac{\delta}{2\left[\frac{\sinh (\omega h)}{\omega^{2}}-\frac{h}{\omega} \cosh (\omega h)\right]}, \quad \omega^{2}=\frac{C_{44}}{A_{122122}}
$$

and $C_{44}$ is the shear modulus of the classical continuum, $A_{22}=A_{122122}$ is the higher order modulus of strain gradient elasticity law, see (12). These functions will be used for comparison to finite element computations in the following sections.

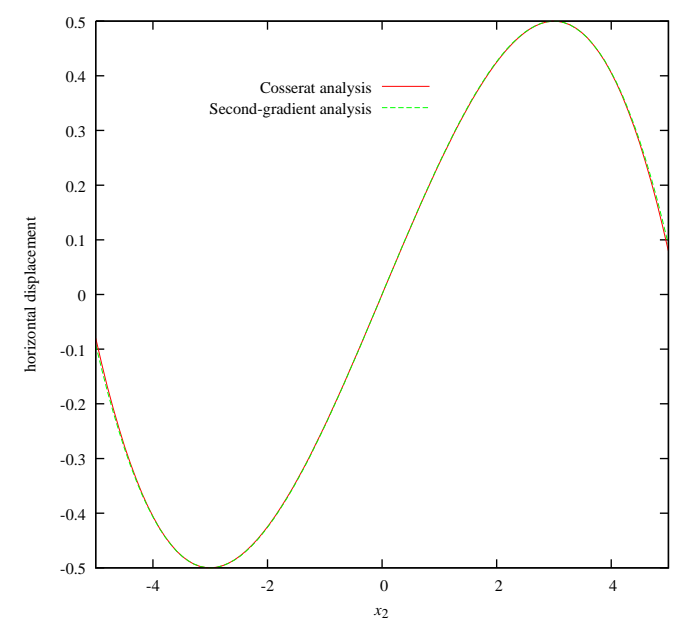

Fig. 3. Cosserat and second-gradient model analysis of double shear problem. Displacement and coordinate are given in $\mathrm{mm}$ for a set of material parameters given in Table 3

In this simple double shear problems, the higher order elastic moduli can be adjusted in such a way that the Cosserat and second gradient theory provide the same displacement profile $u(x)$ as shown in Fig. 3 . 


\section{DETERMINATION OF ELASTIC MODULI OF COSSERAT AND SECOND GRADIENT HOMOGENEOUS SUBSTITUTION MEDIA}

\subsection{General methodology}

We indicate here how the elastic moduli appearing in the constitutive matrix of equation (9) can be identified from finite element simulations on a unit cell of the material. The unit cell is considered as a heterogeneous Cauchy material. The linear elastic properties of the constituents are assumed to be known. The first step consists in identifying the effective elastic properties of the Cauchy effective medium. This is done by successively prescribing three components of the overall deformation tensor $E_{11}=e_{11}=1, E_{22}=e_{22}=1$ and $E_{12}=\left(e_{12}+e_{21}\right) / 2=0.5$, while keeping vanishing overall relative rotation and curvature. This is the same procedure as in standard periodic homogenization.

The next step consists in applying various combinations of mean strain and relative rotation, as indicated in Table 1 (cases 4,5 and 6 ), while the remaining strain and curvature components are maintained to zero. The anisotropic bending moduli are finally identified by prescribing unit curvature in the absence of overall deformation.

Table 1. Loading cases for the identification of overall Cosserat moduli

\begin{tabular}{|c|c|c|c|c|c|c|c|c|}
\hline Moduli & $Y_{1111}$ & $Y_{2222}$ & $Y_{1122}$ & $Y_{1212}$ & $Y_{1221}$ & $Y_{2121}$ & $C_{3131}$ & $C_{3232}$ \\
\hline Coefficients & $E_{11}^{2}$ & $E_{22}^{2}$ & $2 E_{11} E_{22}$ & $e_{12}^{2}$ & $2 e_{12} e_{21}$ & $e_{21}^{2}$ & $K_{31}^{2}$ & $K_{32}^{2}$ \\
\hline Case 1 & 1 & 0 & 0 & 0 & 0 & 0 & 0 & 0 \\
\hline Case 2 & 0 & 1 & 0 & 0 & 0 & 0 & 0 & 0 \\
\hline Case 3 & 1 & 1 & 2 & 0 & 0 & 0 & 0 & 0 \\
\hline Case 4 & 0 & 0 & 0 & 1 & 0 & 0 & 0 & 0 \\
\hline Case 5 & 0 & 0 & 0 & 0 & 1 & 0 & 0 & 0 \\
\hline Case 6 & 0 & 0 & 0 & 1 & 2 & 1 & 0 & 0 \\
\hline Case 7 & 0 & 0 & 0 & 0 & 0 & 0 & 1 & 0 \\
\hline Case 8 & 0 & 0 & 0 & 0 & 0 & 0 & 0 & 1 \\
\hline
\end{tabular}

The loading conditions of Table 1 are enforced by prescribing the suitable values of the coefficients in the polynomials (1) to (3). The relations between the coefficients of the polynomials and the macroscopic generalized strain measures depend on the choice of the type of fluctuations $\underline{\boldsymbol{v}}$ in (4). For vanishing or periodic fluctuation $\underline{\boldsymbol{v}}$, direct relations are derived from appropriate averaging rules. They are given in [11] and [12] respectively. We do not recall them here for the sake of brevity. A method for the exact determination of $\underline{\boldsymbol{v}}$ was proposed in $[12,25,26]$ which can lead to slightly different overall moduli. We use here the moduli determined by the latter procedure and the reader is referred to [25] for the detailed identification procedure.

\subsection{Application to a special composite material}

A special periodic two-phase composite material is considered having the unit cell of Fig. 4(a). The edge length of the square unit cell is $l=1 \mathrm{~mm}$. The dark phase is 10000 stiffer that the inclusion grey phase. They are assumed to be isotropic. The respective Young's moduli are $100000 \mathrm{MPa}$ and $10 \mathrm{MPa}$. Both phases have the same Poisson ratio $\nu=0.3$. The volume fraction of the hard phase is $f=23.2 \%$. Note that the microstructure 


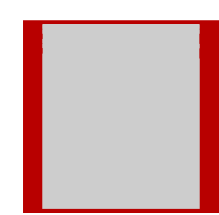

(a)

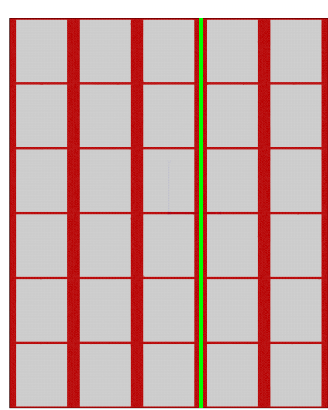

(b)

Fig. 4. Unit cell of the considered periodic composite material (left), structure made of $5 \times 6$ cells to be subjected to various loading conditions (right). The displacement values after deformation will be given along the green line indicated on the structure

exhibits two orthogonal symmetry planes which will lead to orthotropic elastic symmetry. The Cauchy effective elastic moduli are given in Table 2.

Table 2. Elastic properties of the Cauchy homogeneous effective medium

\begin{tabular}{|c|c|c|c|}
\hline$C_{11}(\mathrm{MPa})$ & $C_{12}(\mathrm{MPa})$ & $C_{22}(\mathrm{MPa})$ & $C_{44}(\mathrm{MPa})$ \\
\hline 4939 & 465 & 22066 & 17 \\
\hline
\end{tabular}

The elastic Cosserat and second gradient effective moduli where determined for this material under plane strain condition in [25]. The Cosserat overall moduli are given in Table 3. The analytical solution of the considered double shear problem requires the

Table 3. Elastic properties of the Cosserat homogeneous substitution medium

\begin{tabular}{|c|c|c|c|c|}
\hline $\begin{array}{c}Y_{1212} \\
(\mathrm{MPa})\end{array}$ & $\begin{array}{c}Y_{1221} \\
(\mathrm{MPa})\end{array}$ & $\begin{array}{c}Y_{2121} \\
\left(\mathrm{MPa}_{\mathrm{mm}}{ }^{2}\right)\end{array}$ & $\begin{array}{c}C_{3131} \\
\left(\mathrm{MPa}_{\mathrm{mm}}{ }^{2}\right)\end{array}$ & $\begin{array}{c}C_{3232} \\
\left(\mathrm{MPa}_{2} \mathrm{~mm}^{2}\right)\end{array}$ \\
\hline 51203 & -73977 & 106985 & 803 & 3858 \\
\hline
\end{tabular}

knowledge of the moduli $Y_{1212}, Y_{1221}, Y_{2121}$ and $C_{3232}$. In the case of a second gradient effective medium, we simply need the values of $C_{44}$ (see Table 2) and the higher order modulus

$$
A_{22}=11668 \mathrm{MPa} \cdot \mathrm{mm}^{2}
$$

from the matrix (12). The method of identification of this modulus is explained in [25].

\section{VALIDATION AND LIMITATIONS OF THE GENERALIZED CONTINUUM HOMOGENIZATION METHOD}

Validation of the higher order homogenization is performed first by comparing the results of a reference finite element computation of a heterogeneous structure under double shear external loading conditions and the predictions by means of the analytical solution 
for the overall Cosserat and second gradient substitution media. Then, a more complex loading condition is considered and the Cauchy and Cosserat responses obtained by the finite element method are again compared with the reference numerical solution.

\subsection{Reference computations}

The considered composite structure is made of $5 \times 6$ unit cells, as shown in Fig. 4(b). Opposite horizontal displacement and vanishing vertical displacement conditions are applied at the bottom and top edges of the structure, as in Fig. 2(a). The deformed state of this structure is shown in Fig. 5(a). We will also consider the loading condition of Fig.

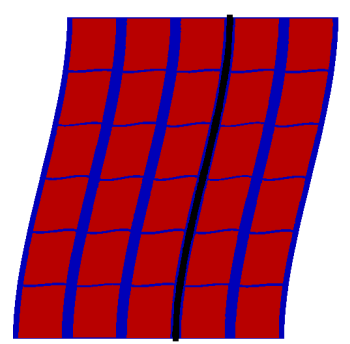

(a)

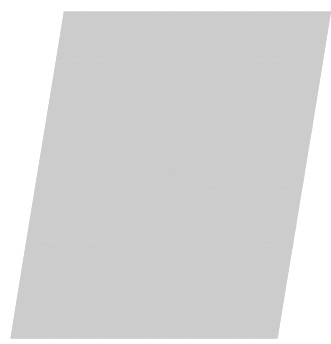

(b)

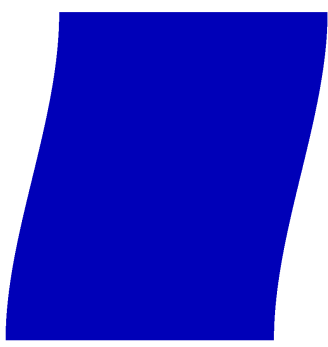

(c)

Fig. 5. Deformed shapes of the $5 \times 6$ cell structure subjected to double shear with clamping conditions based on finite element simulations: (a) Reference computation, (b) use of a Cauchy homogeneous effective medium, (c) use of a Cosserat homogeneous substitution medium

2 (b) that combines bending and shear. The bottom edge is fixed whereas the top edge is rotated by $30^{\circ}$ with respect to the geometric center of the the structure. The corresponding deformed state is shown in Fig. 6(a). They are obtained by the finite element method within

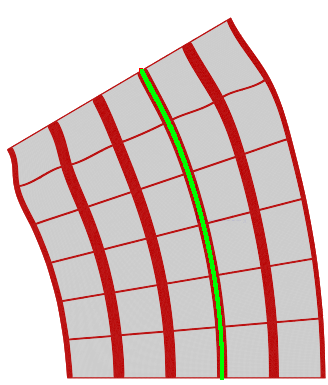

(a)

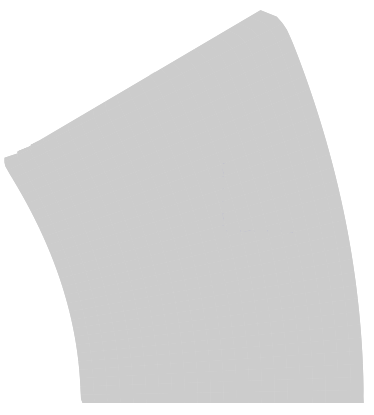

(b)

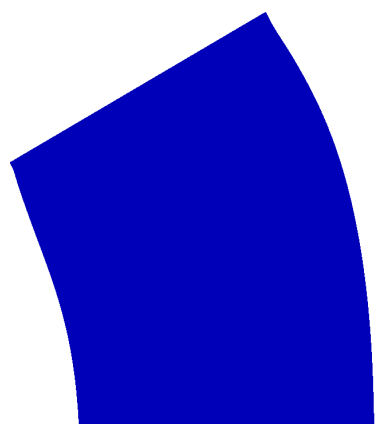

(c)

Fig. 6. Deformed shapes of the $5 \times 6$ cell structure subjected to combined bending - shear loading conditions: (a) Reference computation, (b) use of a Cauchy homogeneous effective medium, (c) use of a Cosserat homogeneous substitution medium

the context of linear elasticity. 


\subsection{Comparison of Cauchy, Cosserat and second gradient predictions}

For both loading conditions we compare the responses of the homogeneous equivalent Cauchy, Cosserat and second gradient media to the reference finite element computations. Finite element simulations have been performed for the overall Cauchy and Cosserat media. Regarding the overall second gradient medium, the analytical prediction (28) is considered for comparison in the case of double shear.

The deformed states of the Cauchy and Cosserat homogeneous structures are shown in Fig. 5 and 6(b) and (c). In the double shear problem, the response of the Cauchy structure is entirely dominated by shear deformation contrary to the reference structure which experiences significant bending at the upper and lower ends. The bending is accounted for by the Cosserat structure due to the fact that additional boundary conditions were prescribed at the top and bottom surfaces, namely vanishing microrotation $\Phi_{3}$ for clamping conditions in analogy to a Timoshenko beam problem.

The Cosserat medium also better reproduces the general shape of the structure deformed by the more complex loading condition of Fig. 6(c). We have applied the extra boundary conditions $\Phi_{3}=0$ at the bottom and $\Phi_{3}=30^{\circ}$ at the bottom. In particular the slight bump on the lateral left edge is captured by the Cosserat model but not by the Cauchy model.

More quantitative comparison is possible by plotting the displacement component $U_{1}$ along the vertical line displayed on the structure of Fig. 4(b). The curves of Fig. 7(a) confirm that the double shear induced combined shear and bending which is perfectly

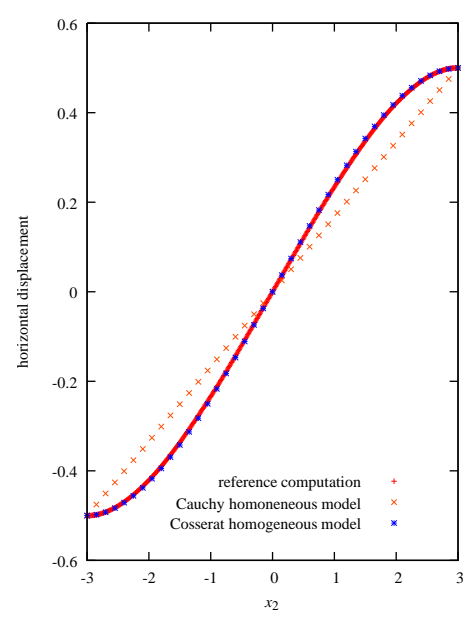

(a)

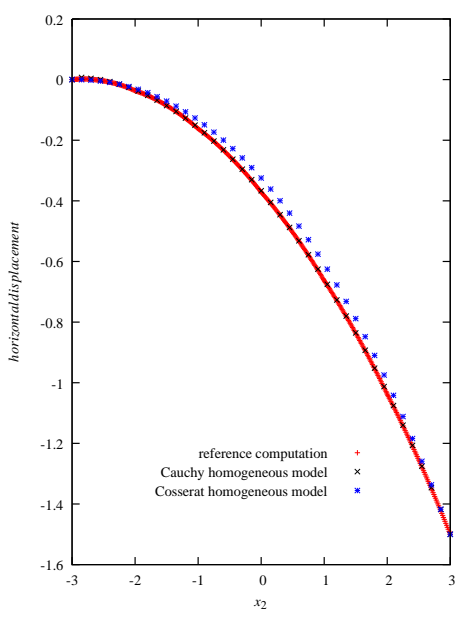

(b)

Fig. 7. Displacement profile along the vertical line indicated in Fig. 4: Double shear loading (a) and rotation loading (b)

reproduced by the Cosserat medium and not at all by the homogeneous equivalent Cauchy material. 
Note that the second gradient model also provides an excellent estimate of the displacement profile, without visible difference from the Cosserat prediction. This may be surprising insofar as the second gradient bending modulus $A_{22}$ was found to be significantly larger than the Cosserat bending modulus $C_{3232}$.

In contrast, the curves of Fig. 7(b) show that both the Cosserat and Cauchy continua correctly reproduced the horizontal displacement along the vertical line, although the Cosserat response is slightly less satisfactory under these conditions. The curves of Fig. 8 clearly show the improvement brought by the additional boundary conditions in

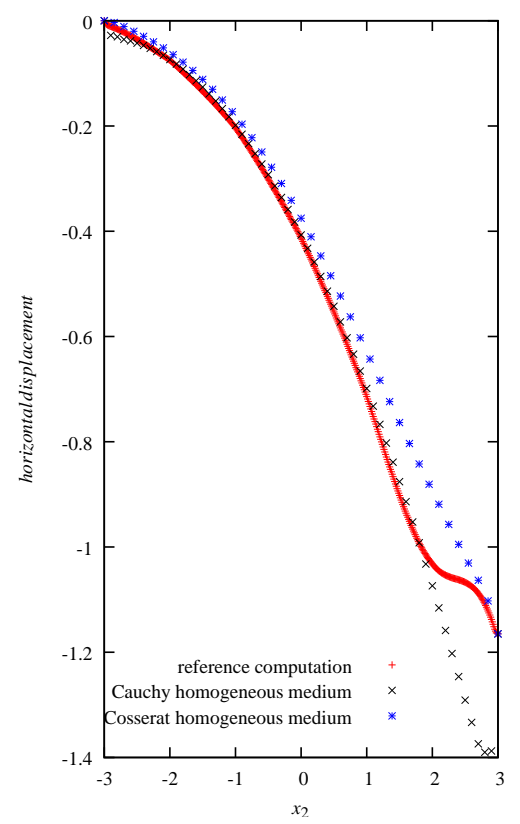

Fig. 8. Horizontal displacement of left boundary of the $5 \times 6$ cell structure subjected to rotation loading with clamping conditions based on finite element simulations of Fig. 6

the Cosserat continuum even though the displacement far from the boundary is better described by the Cauchy continuum than by the Cosserat medium.

\section{CONCLUSIONS}

Analytical and finite element results have been provided to illustrate the capability of generalized continuum theories to better capture the overall response of composite structures than the Cauchy continuum. This happens to be the case when the composite structure is subjected to severe macroscopic strain gradients which arise due to the fact that the size of the heterogeneities is not small enough compared to the structural dimensions. In the case of double shear loading, both the second gradient and Cosserat media were able to capture the structural response. More complicated loading conditions must be worked out in order to evidence the specific features of these distinct theories. 
Several open questions remain to be solved before higher order homogenization can be considered as a settled problem. In particular, one should expect that a Cauchy effective medium is retrieved when the contrast in the elastic properties of the constituent is small. This is indeed the case if one follows the definitions of effective Cosserat and strain gradient elastic properties proposed by [3] and [21]. One may also expect that the effective higher order moduli should not depend on the specific choice of the periodic unit cell. According to the procedure proposed by [21], the effective higher order properties will indeed not depend on the number of unit cells considered $(1 \times 1,2 \times 2, \ldots)$ but cells translated by a vector smaller than the period will lead to slightly different moduli. This deficiency may not be limiting in the sense that different higher order moduli may deliver close predictions, as experienced in the comparison shown in this work between the Cosserat and second gradient substitution media.

It is also necessary to explore new microstructures more prone to strain gradient effects than the considered example in order to illustrate the improvement brought by generalized continuum theories compared to standard homogenization methods. Such suited microstructures may be inspired from the truss constructions considered by [1].

\section{REFERENCES}

[1] Alibert J.J., Seppecher P., and Dell'Isola, Truss Modular Beams with Deformation Energy Depending on Higher Displacement Gradients, Mathematics and Mechanics of Solids, 8, (2003), 51-73.

[2] Bacigalupo A. and Gambarotta L., Second-order computational homogenization of heterogeneous materials with periodic microstructure, ZAMM, 90, (2010), 796-811.

[3] Bigoni D. and Drugan W.J., Analytical derivation of Cosserat moduli via homogenization of heterogeneous elastic materials, Journal of Applied Mechanics, 74, (2007), 741-753.

[4] Boutin C., Microstructural effects in elastic composites, Int. J. Solids Structures, 33, (1996), 1023-1051.

[5] Enakoutsa K. and Leblond J.B., Numerical implementation and assessment of the GLPD micromorphic model of ductile rupture, European Journal of Mechanics A/solids, 28, (2009), 445-460.

[6] Feyel F., A multilevel finite element method (FE2) to describe the response of highly nonlinear structures using generalized continua, Comp. Meth. Appl. Mech. Engng, 192, (2003), 3233-3244.

[7] Forest S., Mechanics of Generalized Continua : Construction by Homogenization, Journal de Physique IV, 8, (1998), Pr4-39-48.

[8] Forest S., Aufbau und Identifikation von Stoffgleichungen für höhere Kontinua mittels Homogenisierungsmethoden, Technische Mechanik, 19(4), (1999), 297-306.

[9] Forest S., Pradel F., and Sab K., Asymptotic analysis of heterogeneous Cosserat media, International Journal of Solids and Structures, 38, (2001), 4585-4608.

[10] Forest S. and Sab K., Cosserat Overall Modeling of Heterogeneous Materials, Mechanics Research Communication, 25, (1998a), 449-454.

[11] Forest S. and Sab K., Cosserat Overall Modeling of Heterogeneous Materials, Mechanics Research Communications, 25(4), (1998b), 449-454.

[12] Forest S. and Trinh D. K., Generalized continua and non-homogeneous boundary conditions in homogenization methods, ZAMM, 91, (2011), 90-109. 
[13] Geers M.G.D., Borst R. de, Brekelmans W.A.M, and Peerlings R.H.J., Validation and internal length scale determination for a gradient damage model: application to short glassfibre-reinforced polypropylene, International Journal of Solids and Structures, 36, (1999), 2557-2583.

[14] Geers M.G.D., Kouznetsova V.G., and Brekelmans W.A.M, Gradient-enhanced computational homogenization for the micro-macro scale transition, Journal de Physique IV, 11, (2001), Pr5-145-152.

[15] Gologanu M., Leblond J.B., and Devaux J., Continuum micromechanics, chapter Recent extensions of GursonÒs model for porous ductile metals, Springer Verlag, CISM Courses and Lectures, 377, 61-130.

[16] Jänicke R. and Diebels, A numerical homogenisation strategy for micromorphic continua, Nuovo Cimento della Societa Italiana di Fisica C-Geophysics and Space Physics, 32, (2009), 121-132.

[17] Jänicke R., Diebels S., Sehlhorst H.-G., and Düster A., Two-scale modelling of micromor-phic continua, Continuum Mechanics and Thermodynamics, 21, (2009), 297-315.

[18] Kouznetsova V., Geers M. G. C., and Brekelmans W. A. M., Size of a RVE in a second order computational homozenization framework, International Journal for Multiscale Computational Engineering, 2, (2004a), 575-598.

[19] Kouznetsova V. G., Geers M. G. D., and Brekelmans W. A. M., Multi-scale second-order computational homogenization of multi-phase materials : A nested finite element solution strategy, Computer Methods in Applied Mechanics and Engineering, 193, (2004b), 5525-5550.

[20] Kruch S. and Forest S., Computation of Coarse Grain Structures Using a Homogeneous Equivalent Medium, Journal de Physique IV, 8, (1998), Pr8-197-205.

[21] Li J., Establishment of strain gradient constitutive relations by homogenization, Comptes Rendus Mécanique, 339, (2011), 235-244.

[22] Liu X. and $\mathrm{Hu}$ G, Inclusion problem of microstretch continuum, International Journal of Engineering Science, 42, (2003), 849-860.

[23] Mindlin R.D. and Eshel N.N., On first strain gradient theories in linear elasticity, Int. J. Solids Structures, 4, (1968), 109-124.

[24] Sanchez-Palencia E. and Zaoui A., Homogenization techniques for composite media, Lecture Notes in Physics, Springer, Berlin, 272, (1987).

[25] Trinh D. K., Jänicke R., Auffray N., Diebels S., and Forest S., Evaluation of generalized continuum substitution models for heterogeneous materials, International Journal of Multiscale Computational Engineering, (2011) (accepted).

[26] Trinh K. D. and Forest S., The role of the fluctuation field in higher order homogenization, PAMM Proc. Appl. Math. Mech., 10, (2010), 431-432.

[27] Trovalusci P. and Masiani R., Non-linear micropolar and classical continua for anisotropic discontinuous materials, International Journal of Solids and Structures, 40, (2003), 12811297.

[28] Xun F., Hu G., and Huang Z., Size-dependence of overall in-plane plasticity for fiber composites, International Journal of Solids and Structures, 41, (2004), 4713-4730.

[29] Yuan X., Tomita Y., and Andou T., A micromechanical approach of nonlocal modeling for media with periodic microstructures, Mechanics Research Communications, 35, (2008), 126133. 